\title{
A do-it-yourself protocol for simple transcription activator-like effector assembly
}

\author{
Claudia Uhde-Stone ${ }^{1}$, Nilang Gor ${ }^{1,2}$, Tiffany Chin², Joseph Huang ${ }^{2}$ and Biao Lu²
}

\begin{abstract}
Background: TALEs (transcription activator-like effectors) are powerful molecules that have broad applications in genetic and epigenetic manipulations. The simple design of TALEs, coupled with high binding predictability and specificity, is bringing genome engineering power to the standard molecular laboratory. Currently, however, custom TALE assembly is either costly or limited to few research centers, due to complicated assembly protocols, long set-up time and specific training requirements.

Results: We streamlined a Golden Gate-based method for custom TALE assembly. First, by providing ready-made, quality-controlled monomers, we eliminated the procedures for error-prone and time-consuming set-up. Second, we optimized the protocol toward a fast, two-day assembly of custom TALEs, based on four thermocycling reactions. Third, we increased the versatility for diverse downstream applications by providing series of vector sets to generate both TALENs (TALE nucleases) and TALE-TFs (TALE-transcription factors) under the control of different promoters. Finally, we validated our system by assembling a number of TALENs and TALE-TFs with DNA sequencing confirmation. We further demonstrated that an assembled TALE-TF was able to transactivate a luciferase reporter gene and a TALEN pair was able to cut its target.

Conclusions: We established and validated a do-it-yourself system that enables individual researchers to assemble TALENs and TALE-TFs within 2 days. The simplified TALE assembly combined with multiple choices of vectors will facilitate the broad use of TALE technology.
\end{abstract}

Keywords: TALEN, TALE-TF, Golden Gate, Transcription-activator-like effector

\section{Background}

With the recent emergence of transcription activator-like effector (TALE) technology, gene editing has entered an exciting new era [1-3]. While zinc finger nucleases have been well established for the purpose of generating targeted mutations [4], their challenging design and need for experimental optimization have restricted this technology to few, highly specialized laboratories. In contrast, TALEs are simple to design, able to target almost any DNA sequence within the genome, and promise less off-target effects compared to zinc-finger nucleases [5-7].

Native TALEs are transcription factors used by plantpathogenic bacteria in the genus Xanthomonas. They activate transcription of host genes by binding to specific sequences in the promoter region of the targeted gene $[8,9]$. Strikingly, the TALE DNA binding domain consists

\footnotetext{
* Correspondence: BLu@systembio.com

${ }^{2}$ System Biosciences (SBI), 265 North Whisman Rd, Mountain View, CA 94043, USA Full list of author information is available at the end of the article
}

of tandem 33-35 amino acid repeats, followed by a single half repeat of 20 amino acids. Interestingly, the tandem repeats are nearly identical, except for two amino acid codons at position 12 and 13, referred to as "repeatvariable di-residue" (RVD). Each of the four most common RVDs specifies the binding to one of the four nucleotide bases [10,11]. Taking advantage of the simplicity of the TALE coding principle, customized TALEs can be easily designed to allow genetic and epigenetic manipulation. For example, the TALE DNA-binding domains can be combined with either a catalytic DNA endonuclease domain, such as FokI, to allow gene editing, or a transcription factor (TF) domain for gene activation. Indeed, both TALEN and TALE-TF, have been successfully applied to gene-editing or activation in a number of species [2,12-16].

Because of the repetitive nature of the DNA binding domain, the assembly of customized TALEs by direct synthesis or traditional cloning is expensive and technically challenging. Realizing the potential of TALE technology, a

\section{Biomed Central}

(c) 2013 Uhde-Stone et al.; licensee BioMed Central Ltd. This is an Open Access article distributed under the terms of the Creative Commons Attribution License (http://creativecommons.org/licenses/by/2.0), which permits unrestricted use, distribution, and reproduction in any medium, provided the original work is properly cited. 


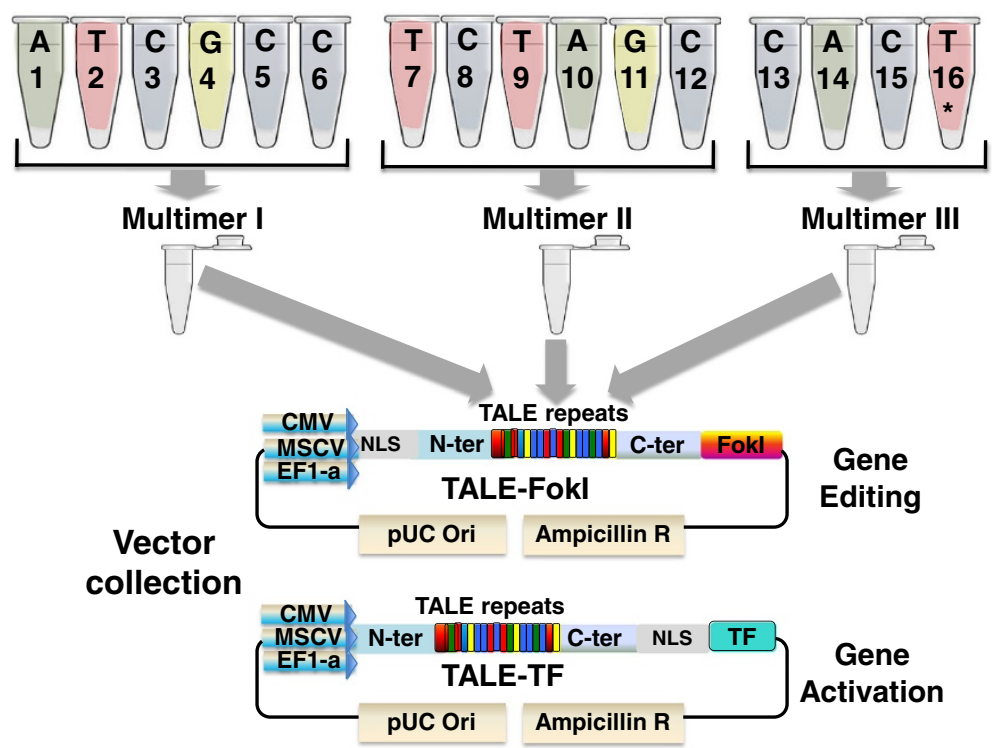

Figure 1 Schematic overview of multimer assembly from the ready-made monomer library into a vector of choice for gene editing or gene activation.

number of approaches for TALE assembly have been devised to allow low to medium throughput $([5,17])$, or high-throughput with automation $[18,19]$. It is worthy to note that these methods are based on the Golden Gate procedure, a cloning strategy that makes use of type IIs restriction enzymes which cut sequences adjacent, rather than within their recognition sites, and allows seamless ligation of repetitive sequences in a specific order $[5,17]$. The Golden Gate cloning technique has proven powerful, but typically relies on the use of large numbers of plasmids or amplified monomers, making this strategy not feasible for the individual research lab.

The objective of this research was to establish a system for simple TALE assembly, and to develop vector sets with gene editing or gene activation capability under the control of different promoters, to allow for a variety of downstream applications.

\section{Results and discussion}

We established a do-it-yourself system for the fast and simple assembly of TAL-repeats into a collection of vectors for TALEN and TALE-TF expression in mammalian cells (Figure 1). This system simplifies the assembly of custom TALEs in three main ways. First, by eliminating the need for time-consuming and error-prone set-up, it shortens time, effort and cost of assembly. Second, by providing a streamlined protocol, it simplifies an otherwise complicated approach, making it feasible for any standard molecular lab. And lastly, a collection of backbone vectors with TALEN and TALE-TF domains under the control of various promoters offers choices for diverse downstream applications in the mammalian system. Using this approach, individual researchers can assemble one or several TALEs into a vector of choice in just 2 days (Figure 2) by using standard molecular techniques and a thermocyler.

The assembly is based on the Golden Gate method, which relies on the ability of type IIS restriction enzymes to cut outside of their recognition site. Type IIS recognition sites arranged in inverse orientation at the $5^{\prime}$ and $3^{\prime}$

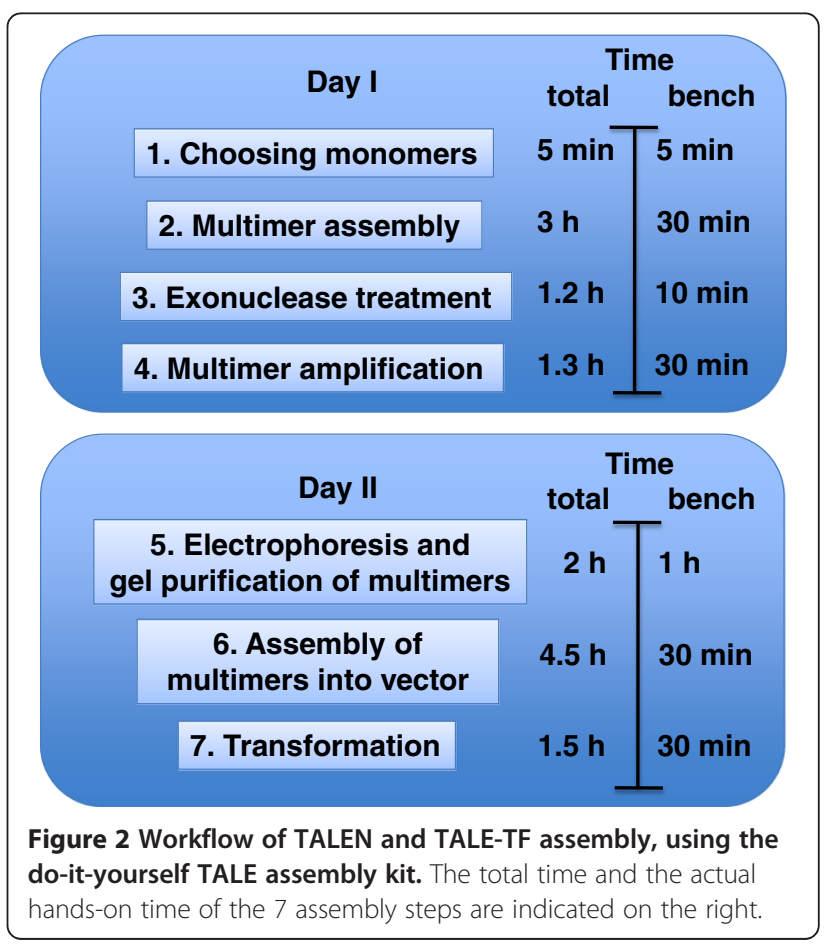


end of a DNA fragment will be removed upon cleavage, allowing simultaneous restriction and ligation. The continuous re-digestion of unwanted ligation products increases the formation of the desired construct. As type IIS fusion sites can be designed to have different sequences, Golden Gate cloning enables directional and seamless assembly of multiple DNA fragments.

As a first step of our do-it-yourself protocol, we assembled monomers into multimers (Figure $3 \mathrm{~A}, \mathrm{~B}$ ), using a procedure based on restriction, ligation and amplification. Multimer 1 and 2 are designed to be hexamers, but the length of multimer 3 can vary to allow variations in the final length, such as 14-19 bp binding sequences. To remove the incompletely assembled and thus linear ligation products, DNA exonuclease treatment was carried out after the multimer assembly. The correctly assembled circular multimers were subsequently amplified by PCR.

On day 2, gel-purified multimers were assembled into a vector of choice, using a second restriction-ligation-based procedure, followed by bacterial transformation (Figure 3C). Colony PCR was performed for confirmation of insert size (Figure 3D); typically, 40-90\% of colonies displayed correct insert size. We recommend to use two colonies of correct insert size for sequence confirmation (Figure 3E, F); typically $80-90 \%$ of sequences revealed correct assembly. A detailed protocol of this approach is provided in the Materials and Methods section.

We have successfully assembled a number of custom TALEs into various vectors. To examine functionality of an assembled TALE, we performed a co-transfection experiment in human embryonic kidney 293 cells. Consistent with previous findings [20], the custom-assembled TALE-TF activated luciferase activity 65 -fold, validating its functionality (Figure 3G, H).

To confirm functionality of an assembled TALEN, we performed a transfection experiment in human embryonic kidney 293 cells using a pair of assembled TALENs to target the AAVS1 locus in the human genome

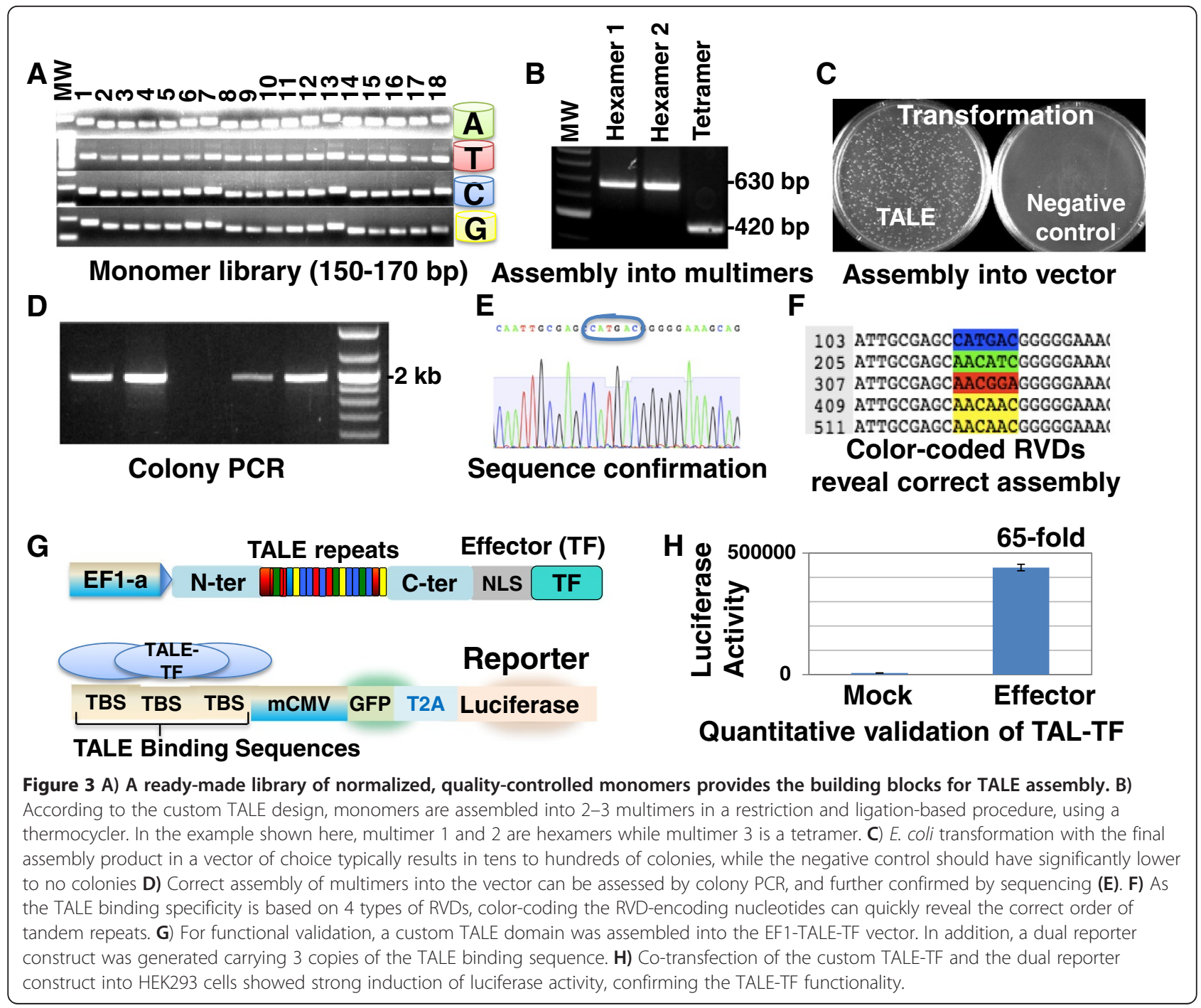


(Figure 4A). For TALEN activity, the Surveyor nuclease mutation detection assay provides a functional validation of successful de novo cutting with a particular pair of TALENs. As shown in Figure 4B, we designed primers that have an amplicon of $650 \mathrm{bp}$ and flanking the centrally localized TALEN target. PCR-amplifcation using this primer pair produced a single band in both mockand TALEN-transfected samples. After denaturing, heteroduplex reannealing and Surveyor nuclease treatment, AAVS1 TALEN-transfected cells displayed an extra band of $\sim 320 \mathrm{bp}$ as predicted (Figure 4C-D). These data validate the function of assembled TALENs using Surveyor nuclease mutation detection assay.

\section{Conclusions}

We established and validated a do-it-yourself strategy that enables researchers to assemble TALE-TF/TALENs in just 2 days. The simplicity of this approach and its minimal hands-on time makes gene-editing an affordable and practical choice for the standard molecular lab. The choices of a number of useful vector sets should further broaden TALE technology to various applications.

\section{Methods}

\section{Experimental design}

Free online tools such as TALEN Targeter [6] and idTALE [21] are available to design TALENs and TALETFs that are specific and have a low risk of off-target effects. TALE-TFs only require one effector protein, while TALENs require the design of protein pairs, which bind two DNA sites, usually spaced 15-30 bp apart to allow for optimal FokI dimerization and cutting [5]. Length of DNA binding sequences may vary, typically ranging from 14-20 bp. In humans, 20 bp may offer high specificity, considering the genome size. It is worthy to note that longer domains (18-20 bp) may decrease cell toxicity by reducing the risk of off-target effects [19].

The appropriate vector can be chosen from a collection of TALEN and TALE-TF backbone vectors, listed in Table 1, according to the desired downstream application. As the last nucleotide of the DNA target is vector

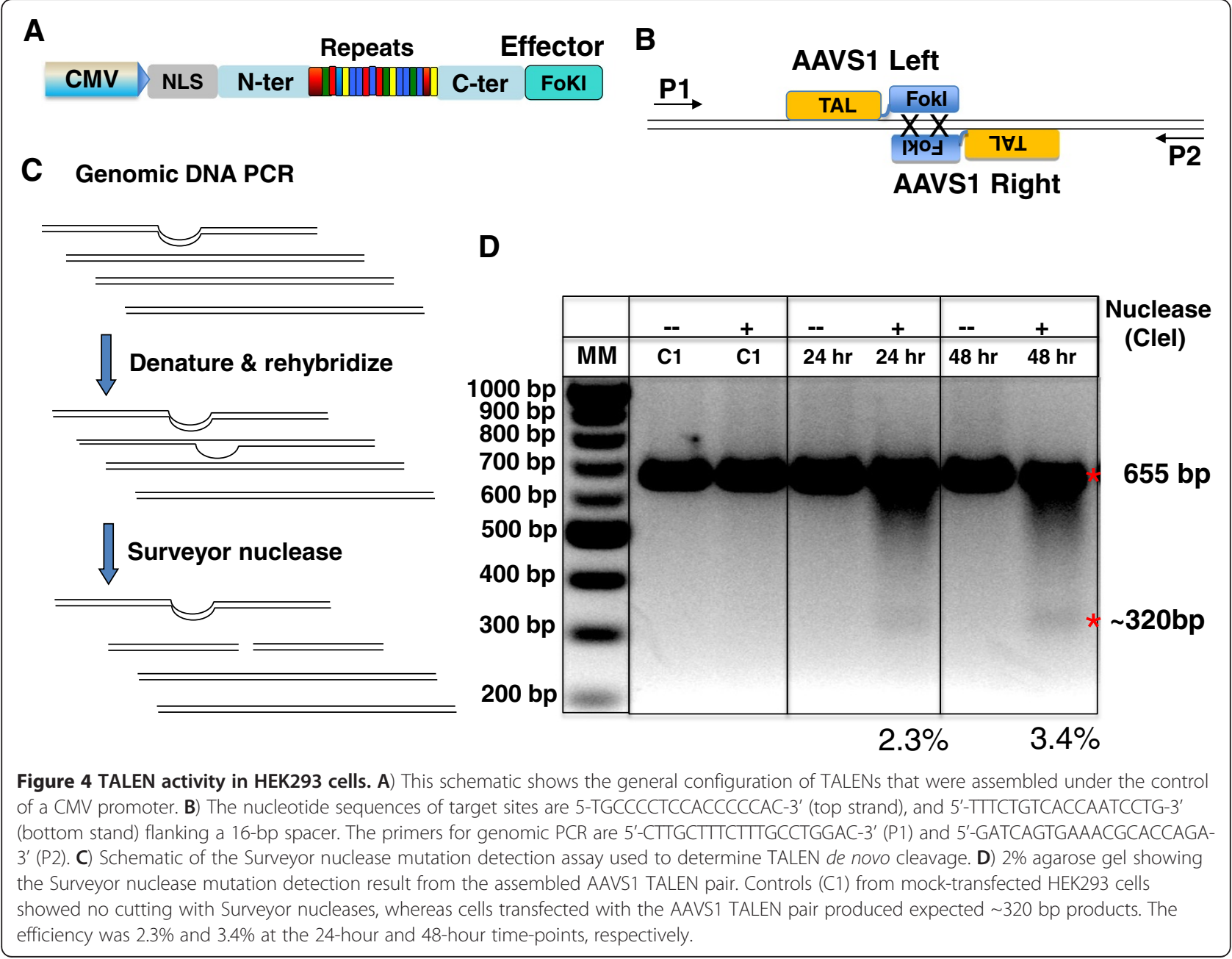


Table 1 Available TALE backbone vectors

\begin{tabular}{|c|c|c|}
\hline Vector $^{1}$ name & Promoter & Application \\
\hline CMV-TALEN-NI & Cytomegalovirus (CMV) & $\begin{array}{l}\text { Targeted } \\
\text { deletions }\end{array}$ \\
\hline \multicolumn{3}{|l|}{ CMV-TALEN-NG } \\
\hline \multicolumn{3}{|l|}{ CMV-TALEN-HD } \\
\hline \multicolumn{3}{|l|}{ CMV-TALEN-NN } \\
\hline EF1-TALEN-NI & Elongation Factor 1-alpha (EF1) & \\
\hline \multicolumn{3}{|l|}{ EF1-TALEN-NG } \\
\hline \multicolumn{3}{|l|}{ EF1-TALEN-HD } \\
\hline \multicolumn{3}{|l|}{ EF1-TALEN-NN } \\
\hline MSCV-TALEN-NI & Murine Stem Cell Virus (MSCV) & \\
\hline \multicolumn{3}{|l|}{ MSCV-TALEN-NG } \\
\hline \multicolumn{3}{|l|}{ MSCV-TALEN-HD } \\
\hline \multicolumn{3}{|l|}{ MSCV-TALEN-NN } \\
\hline CMV-TALE-TF-NI & Cytomegalovirus (CMV) & Targeted \\
\hline \multicolumn{3}{|l|}{ CMV-TALE-TF-NG } \\
\hline \multicolumn{3}{|l|}{ CMV-TALE-TF-HD } \\
\hline \multicolumn{3}{|l|}{ CMV-TALE-TF-NN } \\
\hline EF1-TALE-TF-NI & Elongation Factor 1-alpha (EF1) & \\
\hline \multicolumn{3}{|l|}{ EF1-TALE-TF-NG } \\
\hline \multicolumn{3}{|l|}{ EF1-TALE-TF-HD² } \\
\hline \multicolumn{3}{|l|}{ EF1-TALE-TF-NN } \\
\hline MSCV-TALE-TF-NI & Murine Stem Cell Virus (MSCV) & \\
\hline \multicolumn{3}{|l|}{ MSCV-TALE-TF-NG } \\
\hline MSCV-TALE-TF-HD & & \\
\hline MSCV-TALE-TF-NN & & \\
\hline
\end{tabular}

${ }^{1}$ All vectors come as a set of four, with RVDs NI (A), NG (T), HD (C) or NN (G) specifying the nucleotide binding of the terminal half repeat. ${ }^{2}$ Note that the half-repeat RVD in the EF1-TALE-TF-NG vector is encoded by AAT GGC instead of AAC GGA, and that of the TALE-TF-NN vector is encoded by AAT AAC instead of AACAAC.

encoded, all vectors come in 4 flavors, containing an A, $\mathrm{T}, \mathrm{G}$, or C-specific half repeat.

\section{Materials}

Reagents

EZ-TAL $^{\mathrm{TM}}$ assembly kit (System Biosciences, Mountain View, CA), includes monomer library, Combo Buffer I, Combo Buffer II, BsmBI restriction enzyme, BSaI restriction enzyme, T7 Ligase, Exo buffer, Exonuclease, High-fidelity PCR buffer, High-fidelity DNA polymerase, BSA, DTT, ATP, dNTP, Multimer primer mix, ColonyPCR primer mix, Sequencing primer SF-1 (forward-1), Sequencing primer SF-2 (forward-2), and Sequencing primer SR (reverse).

Additional Reagents not provided in EZ-TAL ${ }^{\mathrm{TM}}$ base kit EZ-TAL ${ }^{\mathrm{TM}}$ end monomers for target size 14-19 (System Biosciences)
Table 2 Components for multimer assembly

\begin{tabular}{ll}
\hline Component & Amount per $\mathbf{1}$ reaction $(\boldsymbol{\mu L})$ \\
\hline Combo Buffer & 1 \\
DTT & 1 \\
ATP & 1 \\
BsmBl restriction enzyme & 0.75 \\
T7 Ligase & 0.25 \\
total & 4 \\
\hline
\end{tabular}

Taq polymerase (New England BioLabs, Ipswich, MA, \#MO267X)

Ultra-Pure Agarose (Life Technologies, Grand Island, NY, \#15510-027)

Agarose Gel Loading Dye Blue (6x) (New England BioLabs, \#B70215)

Quick-Load 2-log DNA Ladder (New England Bio Labs, \#N04695)

1X TAE Electrophoresis Buffer (40 mM Tris, $20 \mathrm{mM}$ acetic acid, and $1 \mathrm{mM}$ EDTA).

Ethidium Bromide (Amresco, Solon, OH, \#X328)

QIAquick Extraction Kit (Qiagen, Valencia, CA, \#28706)

Chemically Competent Escherichia coli, such as One Shot Stbl3 chemically competent E. coli (Life Technologies \# C7373-03)

SOC medium (Life Technologies, \#46-0700)

LB-Carbenicillin Plates (Teknova, Hollister, CA, \#LI008)

LB Broth, (Teknova, \#L8000)

Carbenicillin (Teknova, \#C2130)

\section{Equipment}

Thermocycler (PTC-2000 Peltier Thermal Cycler, MJ Research)

Microfuge tubes (Eppendorf, Hauppauge, NY, 022363204)

Flat-cap PCR tubes (Bio-Rad, Hercules, CA, \#TFI0201)

Micropipetters and tips (Rainin, Columbus, $\mathrm{OH}$ )

Gel electrophoresis equipment for agarose gels (Bio-Rad)

Gel documentation system (ChemiDOC, Bio-Rad)

Incubator for Heat Shock Treatment (Thermomixer R, Eppendorf)

$37^{\circ} \mathrm{C}$ Shaker Model G25 Incubator/Shaker, New Brunswick Scientific)

Culture test tubes with cap (17x100 mm), (Fisherbrand, Houston, TX, \#14-956-6B)

Table 3 Thermocycle conditions for multimer assembly

\begin{tabular}{lll}
\hline Cycle Number & Temperature $\mathbf{1}$ & Temperature 2 \\
\hline 1 & $37^{\circ} \mathrm{C}, 5 \mathrm{~min}$ & \\
$2-16$ & $20^{\circ} \mathrm{C}, 5 \mathrm{~min}$ & $37^{\circ} \mathrm{C}, 5 \mathrm{~min}$ \\
Hold at $4^{\circ} \mathrm{C}$ & & \\
\hline
\end{tabular}


Table 4 Components for exonuclease treatment

\begin{tabular}{ll}
\hline Component & Amount per $\mathbf{1}$ reaction $(\boldsymbol{\mu L})$ \\
\hline Exo buffer & 1.5 \\
ATP & 2 \\
Exonuclease & 1.5 \\
total & 5 \\
\hline
\end{tabular}

\section{Protocol}

TALE assembly

Day 1

1. Choosing monomers (5 min).

Divide the target sequences of 14-20 nucleotides into multimers, excluding the first (5') T and the last (3') nucleotide, which is vector encoded. The first two multimers should be hexamers, the last multimer is variable in size and contains however many monomers remain (excluding the vector-encoded last nucleotide).

\section{Example T | A T C G C C | T C T A G C | C A C $\mathrm{T}^{*} \mid \mathrm{G}$}

Take the corresponding color-coded monomers from the EZ-TAL ${ }^{\mathrm{TM}}$ kit (Figure 1). A special set of "end" monomers can be used to adjust for target sizes shorter than 20 nucleotides (14-19).

2. Multimer assembly (bench time $30 \mathrm{~min}$, total time 3 hours).

In a separate tube for each multimer, pipette $1 \mu \mathrm{L}$ of each monomer; adjust last multimer to $6 \mu \mathrm{L}$ with $\mathrm{H}_{2} \mathrm{O}$ if necessary.

\section{Example}

Multimer 1: $\mathrm{A} 1+\mathrm{T} 2+\mathrm{C} 3+\mathrm{G} 4+\mathrm{C} 5+\mathrm{C} 6$

Multimer 2: $\mathrm{T} 7+\mathrm{C} 8+\mathrm{T} 9+\mathrm{A} 10+\mathrm{G} 11+\mathrm{C} 12$

Multimer 3: $\mathrm{C} 13+\mathrm{A} 14+\mathrm{C} 15+\mathrm{T} 16^{\text {end }}+2 \mu \mathrm{L} \mathrm{H} \mathrm{H}_{2} \mathrm{O}$

Combine components specified in Table 2. Add $4 \mu \mathrm{L}$ of mix to each multimer for a total of $10 \mu \mathrm{L}$. Place each multimer tube in a thermocycler and use cycling conditions specified in Table 3 for $\sim 2.5$ hours.

3. Exonuclease treatment (bench time $10 \mathrm{~min}$, total time 1.2 hours)

To degrade any noncircular ligation products, add components specified in Table 4 .

Table 5 Thermocycle conditions for exonuclease treatment

\begin{tabular}{lll}
\hline Cycle Number & Temperature 1 & Temperature 2 \\
\hline 1 & $37^{\circ} \mathrm{C}, 30 \mathrm{~min}$ & $70^{\circ} \mathrm{C}, 30 \mathrm{~min}$ \\
Hold at $4^{\circ} \mathrm{C}$ & & \\
\hline
\end{tabular}

Table 6 Components for multimer amplification

\begin{tabular}{ll}
\hline Component & Amount per $\mathbf{1}$ reaction $(\boldsymbol{\mu L})$ \\
\hline PCR buffer & 10 \\
DNA-grade Water & 35.5 \\
Multimer primer mix & 2.5 \\
dNTP & 0.5 \\
High-fidelity DNA polymerase & 0.5 \\
total & 49 \\
\hline
\end{tabular}

Add $5 \mu \mathrm{L}$ of master mix to each multimer tube, for a total of $15 \mu \mathrm{L}$. Place each tube in a thermocycler and use cycling conditions specified in Table 5 for 1 hour. 4. Multimer amplification (bench time $30 \mathrm{~min}$, total time 1.3 hours)

To amplify each multimer, combine components specified in Table 6. Add $49 \mu \mathrm{L}$ of mix to $1 \mu \mathrm{L}$ of multimer template each. Perform multimer PCR using conditions specified in Table 7.

\section{Day 2}

5. Gel electrophoresis and purification of multimers (bench time 1 hour, total time 2 hours)

Run all $50 \mu \mathrm{L}$ sample of each amplified multimer in 1 large well or 2 medium-sized wells on a $2 \%$ agarose gel; include a molecular weight marker. Excise multimer bands of correct size. To estimate the correct band size, multiply the number of assembled monomers by 103 bp and add 20 bp. For example, a hexamer will run at about $640 \mathrm{bp}$. When excising the bands, avoid cross-contamination between multimers that are intended for assembly of different TALEs. While bright multimer bands in the range of $20 \mathrm{ng} / \mu \mathrm{L}$ and above are preferred, lower concentrations (10 ng/ $\mu \mathrm{L}$ range) can be assembled, though with lower efficiency. Purify the bands using an appropriate gel purification kit, such as QIAquick Extraction Kit (Qiagen, Valencia, CA) following manufacturer's instructions.

6. Assembly of multimers into vector (bench time 30 min, total time 4.5 hours)

Choose an EZ-TAL ${ }^{\mathrm{TM}}$ backbone vector according to the intended downstream application (see Table 1 for a list of available backbone vectors). The vector should contain the half-repeat that specifies the last

Table 7 Multimer PCR conditions

\begin{tabular}{llll}
\hline Cycle Number & Denature & Anneal & Extend \\
\hline 1 & $95^{\circ} \mathrm{C}, 2 \mathrm{~min}$ & & \\
$2-36$ & $95^{\circ} \mathrm{C}, 20 \mathrm{~s}$ & $60^{\circ} \mathrm{C}, 20 \mathrm{~s}$ & $72^{\circ} \mathrm{C}, 30 \mathrm{~s}$ \\
37 & & & $72^{\circ} \mathrm{C}, 3 \mathrm{~min}$ \\
Hold at $4^{\circ} \mathrm{C}$ & & \\
\hline
\end{tabular}


Table 8 Components for assembly of multimer into vector

\begin{tabular}{lll}
\hline Component & $\begin{array}{l}\text { Amount per } \mathbf{1} \\
\text { reaction }(\boldsymbol{\mu L})\end{array}$ & $\begin{array}{l}\text { Negative } \\
\text { Control }(\boldsymbol{\mu L})\end{array}$ \\
\hline Backbone Vector & 1 & 1 \\
3 Purified Multimers & $5^{*}$ & 0 \\
ATP & 1 & 1 \\
T7 Ligase & 0.25 & 0.25 \\
Combo Buffer II & 1 & 1 \\
BSal enzyme & 0.75 & 0.75 \\
BSA & 1 & 1 \\
DNA-grade $\mathrm{H}_{2} \mathrm{O}$ & 0 & 5 \\
Total & 10 & 10
\end{tabular}

*The proportion of each multimer within these $5 \mu \mathrm{L}$ can be adjusted to achieve a similar concentration of each multimer in the mix.

nucleotide of the target DNA. For example, if the DNA target ends with $\mathrm{T}$, use the $\mathrm{T}$-version of the vector.

Combine the corresponding multimers, vector, and components of the EZ-TAL ${ }^{\mathrm{TM}}$ kit as specified in

Table 8 in a PCR tube for a total of $10 \mu \mathrm{L}$. Include a negative control as shown in Table 8 . If

concentration of multimers is below the recommended range (below $20 \mathrm{ng} / \mu \mathrm{L}$ ), reduce vector concentration accordingly. Place tubes in a thermocycler and use the cycling conditions specified in Table 9.

7. Transformation (bench time $30 \mathrm{~min}$, total time 1.5 hours) Transform competent $E$. coli with the assembly product, following manufacturer's instructions. Plate transformed E. coli on LB with Carbenicillin $(100 \mu \mathrm{g} / \mathrm{L})$; alternatively, Ampicillin $(100 \mu \mathrm{g} / \mathrm{L})$ can be used. It is expected to see tens to hundreds of colonies on plates transformed with the assembled product, while the negative control plates should display significantly fewer or no colonies (Figure 3C).

TALE assembly confirmation Colony PCR (bench time 30 min, total time 2.2 hours) Perform a colony PCR of 5-10 colonies per TALE assembly. For template, slightly dip a sterile pipette tip into a colony, streak an LB plate once to keep track of the colony, and then dip the pipette tip into a tube with $100 \mu \mathrm{L}$ sterile $\mathrm{H}_{2} \mathrm{O}$. For negative control, swipe over agar

Table 9 Thermocycle conditions for assembly of multimers into vector

\begin{tabular}{llll}
\hline Cycle Number & Temperature 1 & Temperature 2 & Temperature 3 \\
\hline 1 & $37^{\circ} \mathrm{C}, 5 \mathrm{~min}$ & & \\
$2-20$ & $20^{\circ} \mathrm{C}, 5 \mathrm{~min}$ & $37^{\circ} \mathrm{C}, 5 \mathrm{~min}$ & \\
21 & & & $80^{\circ} \mathrm{C}, 20 \mathrm{~min}$ \\
Hold at $4^{\circ} \mathrm{C}$ & & & \\
\hline
\end{tabular}

Table 10 Components for colony PCR

\begin{tabular}{ll}
\hline Component & Amount $(\mu \mathrm{L})$ \\
\hline dNTP & 0.2 \\
Colony-PCR primer mix & 0.2 \\
Taq Polymerase $\left(5 \cup \mu \mathrm{L}^{-1}\right)$ & 0.1 \\
Taq Polymerase buffer, 10x & 2 \\
DNA-grade $\mathrm{H}_{2} \mathrm{O}$ & 16.5 \\
total & 19 \\
\hline
\end{tabular}

plate between colonies, and process like a colony dip. Label colony streaks according to tube numbers, and incubate over night at $37^{\circ} \mathrm{C}$.

Set up a colony PCR as specified in Table 10. Add 19 $\mu \mathrm{l}$ of mix to $1 \mu \mathrm{L}$ of diluted template (colony suspension or negative control in $100 \mu \mathrm{L} \mathrm{H} \mathrm{H}_{2} \mathrm{O}$ ) for a total of $20 \mu \mathrm{L}$. Place tubes in thermocycler, and run using cycling conditions as specified in Table 11.

\section{Agarose gel electrophoresis of colony PCR (bench time 30 min, total time 1.5 hours)}

Run all $20 \mu \mathrm{L}$ of the colony-PCR samples and negative control on a $1 \%$ agarose gel; include a molecular weight marker. The expected size of the band can be calculated as number of inserted monomers x 103 bp plus $250 \mathrm{bp}$.

\section{Sequence confirmation}

Inoculate 2 colonies that show a single band of correct size in LB + Carbenicillin $(100 \mu \mathrm{g} / \mathrm{L})$ or Ampicillin (100 $\mu \mathrm{g} / \mathrm{L}$ ), and incubate over night for plasmid isolation. For sequence confirmation, use sequencing primers provided in the EZ-TAL ${ }^{\mathrm{TM}}$ kit, EZ-TAL ${ }^{\mathrm{TM}} \mathrm{SF} 1$ (forward-1), EZ$\mathrm{TAL}^{\mathrm{TM}}$ SF2 (forward 2), and EZ-TAL ${ }^{\mathrm{TM}}$ SR (reverse). For short TALEs with DNA target sites of 14-15 bp, primer EZ-TAL ${ }^{\mathrm{TM}} \mathrm{SF} 2$ can be omitted.

\section{Trouble-shooting advice}

\section{Poor multimer amplification}

A faint or missing multimer band in combination with smear in the high molecular-weight range may indicate failed or impaired exonuclease treatment. Ensure correct storage conditions of exonuclease enzyme and ATP, and make sure that all components are added in correct amounts. Do not skip or shorten exonuclease incubation time.

Table 11 Thermocycle conditions for colony PCR

\begin{tabular}{llll}
\hline Cycle Number & Denature & Anneal & Extend \\
\hline 1 & $94^{\circ} \mathrm{C}, 3 \mathrm{~min}$. & & \\
$2-31$ & $94^{\circ} \mathrm{C}, 30 \mathrm{~s}$ & $60^{\circ} \mathrm{C}, 30 \mathrm{~s}$ & $72^{\circ} \mathrm{C}, 2 \mathrm{~min}$ \\
32 & & & $72^{\circ} \mathrm{C}, 5 \mathrm{~min}$ \\
Hold at $4^{\circ} \mathrm{C}$ & & & \\
\hline
\end{tabular}




\section{Colony PCR does not show bands of correct size}

To assess quality and quantity of the gel-purified multimers, run an aliquot on an agarose gel. Multimers of 10 $\mathrm{ng} / \mu \mathrm{L}$ and less can still be assembled, but with reduced efficiency. If concentration of multimers is lower than recommended (below $20 \mathrm{ng} / \mu \mathrm{L}$ ), reduce vector concentration in the assembly step (step 6) accordingly. If necessary, redo any multimer of low concentration and/or quality.

\section{Sequence error}

About $10-20 \%$ of sequenced clones may display a sequence error, such as an incorrect repeat or a frame shift. In this case, we recommend sequencing some additional clones. If this does not reveal an error-free sequence, we recommend to redo the multimer that contains the error, and re-assemble into the vector. Note that the half-repeat RVD in the EF1-TALE-TF-NG vector is encoded by AAT GGC instead of AAC GGA, and that of the TALE-TF-NN vector is encoded by AAT AAC instead of AACAAC (see Table 1, footnote).

\section{Abbreviations}

C-ter: Carboxy terminus; CMV: Cytomegalovirus promoter; EF1-a: Elongation Factor 1-alpha promoter; GFP: Green Fluorescent Protein; mCMV: minimal CMV promoter; MSCV: Murine Stem Cell Virus promoter; MW: Molecular Weight Marker; NLS: Nuclear localization signal; N-ter: Amino terminus; ORI: origin of replication; R: Resistance; RVD: repeat-variable di-residue; TALE: Transcription activator-like effector; TALEN: Transcription activator-like effector nuclease; TF: transcription factor; T2A: Self-cleaving 2A peptide sequence.

\section{Competing interests}

$\mathrm{BL}, \mathrm{NG}, \mathrm{TC}$ and $\mathrm{JH}$ declare financial competing interest as SBI (System Biosciences) employees. CUS declares competing interest as a collaborator with SBI.

\section{Authors' contributions}

CUS and BL conceived the study aims and design. CUS, BL, NG and TC performed the experiments and interpreted the results. CUS drafted the manuscript. BL, NG, TC and JH contributed to the revision of the manuscript. All authors have read and approved the final version of this paper.

\section{Author details}

'Department of Biological Sciences, California State University, East Bay, 25800 Carlos Bee Blvd, Hayward, CA 94542, USA. ²System Biosciences (SBI), 265 North Whisman Rd, Mountain View, CA 94043, USA.

Received: 3 January 2013 Accepted: 5 January 2013

Published: 14 January 2013

\section{References}

1. Clark KJ, Voytas DF, Ekker SC: A TALE of two nucleases: gene targeting for the masses? Zebrafish 2011, 8:147-149.

2. Hockemeyer D, Wang H, Kiani S, Lai CS, Gao Q, Cassady JP, Cost GJ, Zhang L, Santiago Y, Miller JC, Zeitler B, Cherone JM, Meng X, Hinkley SJ, Rebar EJ, Gregory PD, Urnov FD, Jaenisch R: Genetic engineering of human pluripotent cells using TALE nucleases. Nature biotechnology 2011, 29:731-734.

3. Morbitzer R, Romer P, Boch J, Lahaye T: Regulation of selected genome loci using de novo-engineered transcription activator-like effector (TALE)-type transcription factors. Proceedings of the National Academy of Sciences of the United States of America 2010, 107:21617-21622.

4. Urnov FD, Rebar EJ, Holmes MC, Zhang HS, Gregory PD: Genome editing with engineered zinc finger nucleases. Nature reviews. Genetics 2010, 11:636-646.
5. Cermak T, Doyle EL, Christian M, Wang L, Zhang Y, Schmidt C, Baller JA, Somia NV, Bogdanove AJ, Voytas DF: Efficient design and assembly of custom TALEN and other TAL effector-based constructs for DNA targeting. Nucleic acids research 2011, 39:82.

6. Doyle EL, Booher NJ, Standage DS, Voytas DF, Brendel VP, Vandyk JK, Bogdanove AJ: TAL Effector-Nucleotide Targeter (TALE-NT) 2.0: tools for TAL effector design and target prediction. Nucleic acids research 2012, 40:W117-122.

7. Garg A, Lohmueller JJ, Silver PA, Armel TZ: Engineering synthetic TAL effectors with orthogonal target sites. Nucleic acids research 2012, 50:7584-7595.

8. Boch J, Bonas U: Xanthomonas AvrBs3 family-type III effectors: discovery and function. Annual review of phytopathology 2010, 48:419-436.

9. Bogdanove AJ, Schornack S, Lahaye T: TAL effectors: finding plant genes for disease and defense. Curr Opin Plant Biol 2010, 13:394-401.

10. Boch J, Scholze H, Schornack S, Landgraf A, Hahn S, Kay S, Lahaye T, Nickstadt A, Bonas U: Breaking the code of DNA binding specificity of TAL-type III effectors. Science 2009, 326:1509-1512.

11. Moscou MJ, Bogdanove AJ: A simple cipher governs DNA recognition by TAL effectors. Science 2009, 326:1501

12. Li T, Huang S, Zhao X, Wright DA, Carpenter S, Spalding MH, Weeks DP, Yang B: Modularly assembled designer TAL effector nucleases for targeted gene knockout and gene replacement in eukaryotes. Nucleic acids research 2011, 39:6315-6325.

13. Li T, Liu B, Spalding MH, Weeks DP, Yang B: High-efficiency TALEN-based gene editing produces disease-resistant rice. Nature biotechnology 2012, 30:390-392.

14. Liu J, Li C, Yu Z, Huang P, Wu H, Wei C, Zhu N, Shen Y, Chen Y, Zhang B, Deng WM, Jiao R: Efficient and Specific Modifications of the Drosophila Genome by Means of an Easy TALEN Strategy. Journal of genetics and genomics = Yi chuan xue bao 2012, 39:209-215.

15. Mahfouz MM, Li L: TALE nucleases and next generation GM crops. GM Crops 2011, 2:99-103.

16. Moore FE, Reyon D, Sander JD, Martinez SA, Blackburn JS, Khayter C,

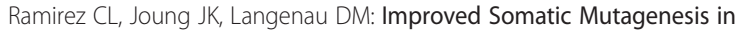
Zebrafish Using Transcription Activator-Like Effector Nucleases (TALENs). Plos one 2012, 7:e37877.

17. Sanjana NE, Cong L, Zhou Y, Cunniff MM, Feng G, Zhang F: A transcription activator-like effector toolbox for genome engineering. Nature protocols 2012, 7:171-192.

18. Briggs AW, Rios $X$, Chari R, Yang L, Zhang F, Mali P, Church GM: Iterative capped assembly: rapid and scalable synthesis of repeat-module DNA such as TAL effectors from individual monomers. Nucleic acids research 2012, 40:e117.

19. Reyon D, Tsai SQ, Khayter C, Foden JA, Sander JD, Joung JK: FLASH assembly of TALENs for high-throughput genome editing. Nature biotechnology 2012, 30:460-465.

20. Uhde-Stone C, Huang J, Lu B: A robust dual reporter system to visualize and quantify gene expression mediated by transcription activator-like effectors. Biological procedures online 2012, 14:8.

21. Li L, Piatek MJ, Atef A, Piatek A, Wibowo A, Fang X, Sabir JS, Zhu JK, Mahfouz MM: Rapid and highly efficient construction of TALE-based transcriptional regulators and nucleases for genome modification. Plant Mol Biol 2012, 78:407-416.

\section{doi:10.1186/1480-9222-15-3}

Cite this article as: Uhde-Stone et al:: A do-it-yourself protocol for simple transcription activator-like effector assembly. Biological Procedures Online 2013 15:3. 